\title{
The Art of War and oncology: applying the principles of strategy and tactics to greater effect in the era of targeted therapy
}

\author{
Emil Lou \\ Department of Medicine, Division of Hematology, Oncology and Transplantation, University of Minnesota, MN, USA \\ Correspondence to: Emil Lou, MD, PhD. Division of Hematology, Oncology and Transplantation, University of Minnesota, 420 Delaware Street SE, \\ Mayo Mail Code 480, Minneapolis, MN 55455, USA. Email: emil-lou@umn.edu.
}

Submitted Feb 14, 2018. Accepted for publication Feb 28, 2018.

doi: 10.21037/atm.2018.03.04

View this article at: http://dx.doi.org/10.21037/atm.2018.03.04

\section{Introduction}

"It is said that if you know your enemies and know yourself, you will not be imperiled in a bundred battles; if you do not know your enemies but do know yourself, you will win one and lose one; if you do not know your enemies nor yourself, you will be imperiled in every single battle."-Sun-Tzu

The rapid and ever accelerating rate of progress in cancer research these past few years has stimulated a great deal of excitement from all quarters: from scientists buoyed by breakthroughs that have elevated our understanding of cancer biology and the effects of the microenvironment; from physicians, now armed with more available and approved therapeutic drugs than ever; and most importantly, from patients and patient advocates who are the most essential stakeholders in this field. This is an historic time for both the clinical practice of oncology and for the field of cancer research as a whole. The moment provides fertile opportunity to reexamine how we view cancer cells, their formation and progression as tumors, and to question previous established paradigms that are now shifting in the current landscape.

In the historic context, over the past 50 years there has been extensive use of analogies of cancer to "war" and "battles" to describe human patients worldwide being treated for and suffering from all forms of this disease. At the legislative level, on December 23, 1971 U.S. President Richard Nixon formed a President's Cancer Panel and signed the National Cancer Act into law, in effect declaring what came to be known more colloquially as the "War on Cancer." That year, an estimated 335,000 Americans died of cancer, and there were an estimated 635,000 new cases (1).
It was believed at that time that this war would be won within 10 years. With the passage of time, with unimagined advances in understanding of the complex biology of cancer, and with the benefit of much hindsight, we have learned that the worldwide War on Cancer is not nearly as simple nor as straightforward as previously thought. The number of cases and deaths from cancer increased considerably over the subsequent 45 years. The total number of American deaths from cancer was estimated at 600,920 in 2017, with $1,688,780$ new cases that year (2). At the patient level, for years many articles and reports in the lay press have seized upon this catchphrase to report on the field of oncology; in recent years, the appropriateness of the War on Cancer analogy has come into question from the medical field (3-6). At the same time, on a personal level—and this is certainly more obvious with the rise of social media use by patient advocates and supportive organizations-the reaction of some patients has been to embrace the analogy and count themselves among an army of "Cancer Warriors" and the like. However, the purpose of this article is actually not to address the War on Cancer analogy at the population or individual level, but rather to apply principles of war strategies and tactics to better understand cancer cell behavior at the cellular and molecular level, with the intent of better identifying patterns of behavior of cancer cells that lead to metastasis and development of treatment resistance in the era of targeted therapy.

Great advances have been made in cancer treatment, especially in molecularly targeted therapies. For example, as cited in the Annual Cancer Progress Report from the American Association for Cancer Research (AACR), between August 2016 and July 2017, the U.S. Food and 
Drug Administration (FDA) approved nine new therapeutic anti-cancer drugs (cancerprogressreport.org). Yet, we are still losing the war against the formidable enemy that is cancer, largely because of the remarkable ability of cancers to adapt and evolve despite improvement in drug development. It is clear that novel strategies and tactics in designing and employing targeted therapeutics are also needed to win this war, and the ability to accomplish this rests squarely on our ability to decipher and react to cancer's ability to evade and evolve around our current arsenal of cancer-directed therapeutic agents.

"Know your enemy and know yourself and you can fight a bundred battles without disaster."

"To know your enemy, you must become your enemy."-Sun-Tzu

Sun-Tzu - an oft-quoted military strategist and general from ancient China-emphasized in his classic tome "The Art of War" that knowledge of one's enemy is essential to designing rational strategic and tactical approaches to war. This book has served for centuries as a guidebook not only for military strategists, but also to people from other fields, including the business world, where it is required reading as much in business schools as it is in military training academies. For those of us in cancer research and clinical oncology, we should be asking whether military lessons from Sun-Tzu and others can be applied to our efforts to understand and better counteract molecular pathways and cellular processes that drive cancer. Can military strategies and tactics such as those outlined in "The Art of War" provide new insights into how to wage a successful campaign against cancer at the biological level? I will provide here several examples in which our approaches to cancer can assimilate and mirror military strategies and tactics to allow us to better understand cancer biology (and its many molecular and physiologic derivatives) and to develop more rational approaches tailored to defeating this powerful enemy.

\section{Tumor heterogeneity: the complexity and composition of tumors undermines current clinical tactics}

Tumor heterogeneity, both within and between solid malignant tumors, is a prime example of a characteristic of malignancy that upsets our notion that one or even a combination of weapons is capable of entirely eliminating cancer. Only in recent years has this topic become more appreciated, mostly because of improvements in technology such as next-generation sequencing and tumor modeling that allow identification of subclone populations that, while potentially small in number, are responsible for propagating tumors over the long-term despite use of aggressive treatments.

In the clinic, oncologists use chemotherapeutic or biologic agents designed to target "the cancer". At least, that is what we tell patients. What we truly mean, with all but a few exceptions, is that we are attempting to target malignant cells of tumors, but are unsure of the ability of these drugs to penetrate tumors with extensive stromatous and other non-malignant components that comprise tumors just as much, if not more so, than malignant cells. Furthermore, we don't yet regularly deploy tactics aimed at breaking down the natural barriers that tumors may construct for themselves. A prime example of this is the dense desmoplastic stromal reaction that pancreatic adenocarcinomas form and which result in a thick and difficult-to-penetrate cloak around tumors. Recent advances have identified novel strategies though. Prominent among them include the use of hyaluronidase, an enzyme that digests hyaluronic acid (HA), a major component of this stromal wall. The ongoing phase III HALO 301 trial incorporates use of hyaluronidase in addition to standardof-care chemotherapeutic agents (gemcitabine and nabpaclitaxel) with intention to simultaneously break down the wall, and thus permit increasing efficacy of drug penetration and presumably increase efficacy (7). Hingorani et al. reported findings from the phase II version of this trial (HALO-109-202) in which patients with tumors that were "HA-high" had a significantly higher progression-free survival (9.2 months) than patients with "HA-low" tumors (5.2 months); overall survival also was higher in patients with HA-high tumors (11.5 months) than in patients with HA-low tumors (8.5 months) (7). Based on that finding, the investigators appropriately reevaluated and revised the therapeutic strategy to incorporate testing of the tumor in its native architectural microenvironment into the screening eligibility guidelines for this trial. This is important because for this type of cancer, biopsy to confirm histopathologic diagnosis is usually obtained via fine needle aspiration obtained during endoscopic ultrasound. Cytology (dispersed cells without preservation of malignant cells in their native microarchitecture) is sufficient to make the diagnosis of cancer. Thus, a trial such as HALO is one example of how studying malignant cells as they are in vivo, in human patients, provides more information that may be used to better strategize and deploy potentially better tactics for treatment. 
Heterogeneity within an individual tumor raises a supremely challenging issue. With molecular medicine advances making their way into clinical pathology laboratories, with more clinically available tests, the particular issue at stake is that these labs will analyze and report the composition based on the specimen they are provided. There is strong potential for selection bias, based on the amount and location of the tumor section biopsied. For this reason, the reported results, when accurate, may be representative of the specimen biopsied, but not necessarily of the tumor as a whole. An example of this challenge has been reported in an elegant study by Sottoriva et al. examining the invasive and highly treatment-resistant malignant brain tumor known as glioblastoma (GB) (8). GB has traditionally been grouped into as many as four histopathologic subtypes. In recent years, with identification of molecular drivers and passengers, the subtypes have been revised to reflect molecular classifications. Sottoriva et al. examined intratumor heterogeneity in GB tumors from human patients (8). Remarkably, 6 of 10 tumors tested harbored multiple subtypes. This is an alarming finding. When the field of neuro-oncology even gets to the point of better tailoring designer drugs to effectively shut down drivers in any given subtype, how will it account for also addressing other potential subtypes that, left untreated, are likely to be responsible for replenishing tumors and take primary responsibility for blocking efficacy of future treatments? One way this is being addressed in systemic [noncentral nervous system (non-CNS)] cancers is through the use of serial biopsies, most prominently in lung cancer using serial assessment of cell-free DNA as a liquid biopsy capable of identifying evolving mutations that mediate resistance $(9,10)$.

The issue of potential heterogeneity between tumors (inter-tumoral heterogeneity) within the same patient has also been investigated in several forms of cancer. The importance of this is underlined by the fact that, for cancers with identifiable druggable targets, there is no firm requirement that the targets be identified in the primary tumor $v s$. in metastatic tumors. There have arguably been no greater cancer-specific advances in identifying effective targeted therapies than in identification of molecular types and biologic drivers of non-small cell lung cancer (NSCLC). In the seminal I-PASS trial published nearly a decade ago $(11,12)$, comparing the use of epidermal growth factor receptor (EGFR)-targeting agent gefitinib vs. standard-ofcare doublet chemotherapy with carboplatin and paclitaxel in Asian patients, subset analyses led to the discovery of a higher proportion of the EGFR mutation in young, female, never-smoking patients with NSCLC. This paradigm has served as an effective example of a "bedside-bench-bedside" paradigm in which a clinical subset that responded well to a drug was effectively studied at both the epidemiological and molecular levels, leading to identification of the EGFR that served as gefitinib's target. Subsequent trials have borne out the targeted therapy approach of only using EGFR inhibitors in patients whose lung tumors harbor the EGFR mutation and avoiding unnecessary use of this class of drugs in patients whose tumors do not harbor an identifiable mutation, thus sparing them unnecessary side effects from an ineffective therapy. After the widespread adoption of gefitinib and similar agents such as erlotinib, the subsequent quandary that arose was the inevitable emergence of resistance to this class of drugs. Again, further lab-based and translational investigations uncovered development of mutations such as T790M that occurred in response to, or emerge in the presence of, EGFR inhibition. Clonal populations that were responsible for reoccurrence and progression of EGFR-driven NSCLC have, in subsequent years, been matched through continual development and trials through the pipeline of $2 \mathrm{nd}, 3 \mathrm{rd}$, and further generations of EGFR inhibitors that have proven to be not only more effective in obtaining objective systemic response, but also in effectively treating NSCLCrelated brain metastasis, a relatively common problem in this cancer that causes substantial morbidity in affected patients. One example of this next-generation evolution is osimertinib, an EGFR-targeting agent that targets T790M mutation-positive NSCLC that was approved by the U.S. FDA in March 2017 for use in the first-line setting $(13,14)$.

While there is potential for tumoral heterogeneity among patients harboring the same tumor subtype, whether in EGFR-mutated NSCLC or other similarly target-driven solid tumor malignancies, there is strong potential for tumors to have or to develop intra-patient heterogeneity. Primary tumors harboring multiple genetic subclones have the capability of "spinning off" metastatic subclones that in some cases bear few significant similarities to the parent tumors. We identify this scenario by referring to tumors as being "discordant" and recognize that this issue has important implications for cancer therapy; yet, this scientific knowledge is not commonly applicable or actionable in the clinical setting. Much of the patient morbidity and mortality in cancer results from complications of metastatic tumor burden. Current treatments often are selected based on the primary tumor, with infrequent assessment of genetic profiles of metastatic tumors that have evolved; we are thus selecting a therapeutic arsenal based on "old" intelligence 
designed to combat a primary tumor that has been superseded by its metastatic clones. Tumor discordancy and heterogeneity allow cancers to perform "evasive maneuvers" to which we are not currently responding sufficiently in the clinical setting. The current wave of clinical trials examining molecularly targeted drugs includes some trials that call for tumor biopsies at intervals during treatment and/or after drug failure, to more intelligently identify causative factors of treatment resistance. While not currently mainstream outside of clinical protocols, these tumor biopsies will provide critical information on tumor heterogeneity over time. Future trials, however, should also include protocols for assessing the totality of tumor heterogeneity at one point in time, to more specifically select treatments capable of killing all subclone populations within a patient. As the actual process of performing biopsy on tumors carries its own inherent risk, the rising specificity and sensitivity of testing circulating tumor cells (CTCs) and circulating cellfree DNA become even more important, with the hope that the "liquid biopsy" approach can become more mainstream and reproducible nationwide as well as worldwide.

The great challenge in this current revolution of bioinformatics and molecular genomics is the fact that our ability to identify potential mutations far outweighs the number of available weapons (i.e., potential drugs) to hit those targets. Recent, ongoing, and future basket trials are designed to determine whether or not drugs that have been identified actually work effectively in patients with tumors harboring those mutations, but that result is not a foregone conclusion. We have the knowledge but remain as of now deficient in the amount of armamentarium at our disposal to use that knowledge.

\section{What to do when tumors perform evasive maneuvers: the lesson that one size does not fit all}

"If I know our troops can attack, but do not know the enemy cannot be attacked, it is only halfway to the victory. If I know the enemy can be attacked, but do not realize our troops cannot attack, it is only halfway to the victory."-Sun-Tzu

We have learned in the targeted therapy era that one size does not fit all-at all. Some types of cancer are in fact composed of groups of molecular subtypes that are different biologically. For example, NSCLC is composed of EGFR, $R O S, A L K, R E T$, and other oncogene-driven subtypes that induce carcinogenesis and which are therapeutic targets. While we do have a better grasp of oncogene addiction- the notion that specific molecular mutations take primary responsibility for driving tumor growth (15) -we have also come to the startling realization that blocking any given molecular driver in one form of cancer does not necessarily work just as well when applied to other cancers. A good example of this is EGFR inhibition in NSCLC (effective) as compared to GB, $40 \%$ of which express a variant of EGFR known as variant III (inhibition not yet shown to be effective). The concept that a targeted agent may work effectively in one type of cancer but not in others with the same target presents a great challenge and lends even greater complexity to tactical approaches to cancer treatment. This knowledge sheds important light on the critical need to improve clinical trial design to address ways to not only block primary driver oncogenes, but also to simultaneously block the alternate molecular pathways that will be upregulated and induce drug resistance. A prime example of this is BRAF, the serine-threonine kinase that is prevalent in more than half of cases of metastatic melanoma. The V600E variant is most common and can be targeted by agents such as vemurafenib. This drug was among the first of a wave of revolutionary strategic approaches to treating a devastating disease. As $5-10 \%$ of colorectal cancers (CRC) also harbor BRAF mutations $(16,17)$, it was reasonable to believe that this drug could work just as well. Preclinical evaluation of vemurafenib in CRC showed significant enough promise to move forward with human trials (18). However, initial efforts at using BRAF inhibitors in metastatic CRC showed no meaningful clinical benefit (e.g., a 5\% response rate) (19), leading cancer researchers back to the drawing board (the lab) to reexamine this problem at the molecular level. The problem was indeed reexamined and feedback activation of EGFR was found to be one culprit (20). This finding was followed by preclinical evaluation of BRAF inhibition using vemurafenib in combination with other chemotherapeutic agents (capecitabine and/or bevacizumab, cetuximab and/ or irinotecan, or erlotinib) (18), and it was found that resistance to BRAF inhibition could be avoided or overcome by also blocking PI3K activation (21). Armed with this insight, translational oncologists applied this understanding to improve trial design, which led to trials examining dual inhibition of BRAF and MEK pathways (22). In the past few years, a cooperative group phase II trial led by Dr. Scott Kopetz through the Southwest Oncology Group (SWOG trial 1406) using a multi-drug regimen of vemurafenib in combination with both irinotecan and cetuximab (23) ultimately showed superior activity and improvement in overall survival. More trials now and in the future need to 
apply this type of strategy in halting both current driving pathways, and concurrently those most likely to become upregulated as cancer's direct response to the drug insult. Once efficacy and tumor response are achieved, it is also imperative to devise next-step strategies to eliminating micrometastatic residual disease with intent to cure. Drs. Bivona and Doebele proposed an elegant blueprint for addressing this important issue in a recent editorial, including tactics aimed at directly addressing tumor genetic heterogeneity and preventing tumor cell adaptation by administering rational polytherapy when warranted, reassessing the role of indefinite $v s$. discontinuation of therapy, and redefining what we should be considering as viable and clinically meaningful objective endpoints in clinical trials (15).

\section{Improving timing: smarter application of available tactics by tailoring timing of treatment administration in the right scenarios}

Some aspects of chemotherapeutic drug administration, sequencing, and timing are already well-established clinically. One example is administration of the commonly used doublet combination of carboplatin and paclitaxel. It is well known at this point that these two drugs have antagonistic interactions; yet, this combination has been and remains a staple of care for treating ovarian and other cancers. What makes the regimen potentially effective in patients is the timing: if carboplatin were to be given after paclitaxel, there would be less total cell-kill; however, if paclitaxel is given first, then the combination works. The antagonistic effect is seen in vitro when both drugs are applied to cancer cells; use of other drugs (e.g., Navitoclax, mimetic of BH3 domain of pro-apoptotic proteins, such as Bax or Bak) can interfere with this antagonistic effect $(24,25)$.

For most cancers, however, we have not yet gotten to the point of examining sequencing or perhaps more important, intervals and timing of drug administration with an eye toward improving efficacy of the drugs independent of dosing and choice of those drugs. Nonetheless, emerging reports have shed light on this aspect, with much of this progress stemming from the emergence of the field of mathematical oncology. To share one intriguing example, again in GB, Leder et al. identified patterns of chemotherapy drug administration concurrent with radiation, which comprises the standard-of-care strategy for treating GB patients after maximal safe surgical resection (26). Their study used theoretical mathematics- based modeling to predict effectiveness of non-standard radiation schedules. While the standard approach clinically is based on clinician availability and patient convenience, it does not take into account targeting maximum biologic effectiveness. Using the approach of Leder et al., they proposed two delivery schedules that they predicted would improve efficacy; tested in vivo in a mouse cancer model, mice treated using this tactic actually had a higher rate of survival.

An even more recent and timely example is found in the world of immunotherapy. More studies are investigating combination strategies using multiple immunotherapeutic drugs (e.g., checkpoint inhibition plus inhibition of CTLA-4). One recent study in mice examined the timing and sequence of administration of anti-PD-1 therapy with an agonist anti-OX40 antibody in a mouse model of breast cancer. The researchers discovered that administration of the former attenuated the effect of the latter when administered concurrently; however, a different and much better efficacy of treatment using these same drugs was seen when antiOX40 treatment was initiated first, followed sequentially by anti-PD-1 (27). This notion that sequence and timing matter has been seen with other drug combinations as well. The vascular endothelial growth factor (VEGF) antagonist bevacizumab is a humanized monoclonal antibody in wide use clinically for many forms of solid tumor malignancies, including metastatic CRC, recurrent GB, NSCLC, and others, most often in combination with cytotoxic chemotherapeutic drugs. It is nearly always used concurrently with chemotherapy, as they were studied in this fashion on clinical trial protocols, presumably due to logistical convenience. The balance between disrupting tumor cell-derived VEGF-mediated angiogenesis, which provides a rich supply of oxygen to hypoxic tumor regions, and the need to prevent inducing leakiness that would diminish effective drug delivery is a tenuous one. For this reason, it is logical to reassess how and in what order we administer chemotherapeutic and biological agents, with the goal of optimizing drug delivery to tumors and maximizing the efficacy of cytotoxic components. One example of this approach is the concept of "preconditioning" tumors with bevacizumab prior to administering chemotherapy. Lu et al. investigated this approach in a single-arm phase II study of 35 patients with brain metastasis secondary to breast cancer, administering two chemotherapeutic agents (etoposide and cisplatin) 24 hours after bevacizumab on the first day of each 21-day cycle (28). The primary endpoint of this study was central nervous system-objective response rate, which 
was $77.1 \%$ overall; $37.1 \%$ of the patients achieved $>80 \%$ volumetric reduction using this approach. Although the study is limited in both number and in being a single-arm trial, the concept can be tested clinically and is consistent with other knowns with the same drug(s) used in variable clinical scenarios. Bevacizumab is routinely used clinically in combination (concurrently) with mono- or doublet-therapy for patients with metastatic (stage IV) CRC, based on data that showed significant improvements in progression-free survival and overall survival, with higher response rates, when including bevacizumab $(29,30)$. However, the same drug combination has shown to have no benefit in stage III patients (i.e., patients who had node-positive disease, but no distant metastasis, and thus underwent surgical resection prior to post-operative therapy) (31), meaning the biologic activity is not just time-dependent, but also dependent on the construction and architecture of the underlying tumor environment. Failures of drugs to work in certain biological contexts, but not in others (based on current standards for assessing success, mainly through measurement of improvement in overall survival) may have a temporal effect and lead us to question whether we are employing the right drugs, but at the wrong times. A fertile timing that is not assessed routinely is use of chemotherapeutic or targeted drugs in the immediate pre- or post-operative period of known invasive malignancies. Is this a lost opportunity to remove residual microscopic disease? For many resected cancers, adjuvant therapy, when indicated, is not administered until weeks or months after surgery, the rationale being that adequate time is needed for patient recovery and optimal wound healing. For patients who heal well within days to weeks, what advantages could be conferred by initiating adjuvant therapy sooner? Few trials have formally investigated this concept in human trials, but one example has been examined using the diabetes drug metformin in breast cancer patients. Based on the rationale that metformin has anti-cancer properties (stimulation of the adenosine monophosphate kinase/AMPK pathway), investigators have applied this drug preoperatively in non-diabetic women with breast cancer in several trials. Tumor specimens from patients in the metformintreatment arms showed significant upregulation of AMPK and downregulation of phospho-Akt (32), and increased apoptosis as well as decreased mitotic rates (33), suggesting biologic activity when using this approach. More novel tactics such as this one are needed to determine whether the lack of systemic treatment of cancers either pre- and/ or post-operatively are compromising our ability to treat patients effectively by permitting invasive cancers time and space to proliferate unchecked, leaving us behind in the race before we even get started.

\section{Understanding the pathway and other aspects of intra- and intercellular communication in cancer: cutting off the enemy's lines of communication}

If one were to devise an effective way to prevent an opponent from sharing information along enemy lines, one such way would be to cut off their lines of communication, whether by radio, phone, instant messaging, satellite, or wireless internet communications. A lack of effective communication has the potential to leave the enemy scattered, disorganized, in disarray, and ultimately ineffective. The correlate in cancer biology is treatment approaches that disrupt internal signaling pathways $(34,35)$. Inhibition of EGFR as described earlier in this text is an excellent example of disrupting a major pathway responsible for driving and promoting growth of a morbid cancer. An emerging field of study at the biological level over the past decade is intercellular communication, the cell-tocell signaling that occurs via various cellular architectural components to, presumably, serve as a method for cellular information sharing. Some forms have been well established for decades-soluble factors, gap junctions, etc. Yet, in practical clinical care, there are few if any available drugs or clinical trials in the pipeline that focus specifically on disrupting and preventing this information sharing. Yet, this strategy represents a potential untapped and missed opportunity. If the communication is so important, and if we can identify the vessels by which these signals are communicated, then theoretically, it is possible to identify drugs that will prevent this process from happening, thus hindering tumor progression. Easier said than done, for sure, but the frontlines of the enemy are no different in requiring synchrony and unity of purpose in order to achieve success.

During the past decade, a unique form of diffusible packaging comprising entities of variable size called exosomes, microvesicles, and microparticles-more broadly known as nano- and micro-sized extracellular vesicles (EVs) - have evolved from being regarded as potential cellular debris of minimal consequence yet detectable in blood, urine, and the tumor microenvironment to being regarded as potential diagnostic/prognostic biomarkers and potential agents of effective drug delivery. These membrane-lined packages, which envelop multivesicular 
bodies extruding from cells, carry cell cargo, such as microRNAs, of clinical relevance due to their ability to fertilize the metastatic niche $(36,37)$ or propagate drug resistance. Thus, the implications of EVs are significant. At the same time, as patterns of diffusion may be somewhat random, it is reasonable to speculate that the quantity of EVs needed to be transmitted to have uptake by at least some cells would need to be high.

In my own laboratory, a central focus of study has been yet another form of cellular communication, one which literally bridges the gap between distant cells in the tumor microenvironment. This intercellular bridge has been referred to by a number of terms, most commonly "tunneling nanotubes (TNTs)", "membrane nanotubes", and "tumor microtubes (TMs)" (38-44). Study of these nanotubes/microtubes is an emerging field of cell biology across a spectrum of disease types. In the past decade, my lab and increasingly others have demonstrated that the cellular bridges not only are found in solid tumors in human patients and in animal models of cancer, but also play important roles in tumor propagation, chemoresistance, and recurrence after treatment (45). TNTs/TMs may figuratively and literally bridge the gap between disparate cells in the post-resection surgical cavity, acting as conduits that facilitate a synchronized re-formation of tumors in the surgical space (45). They may likewise be responsible for coordinating a unified attack of cells at the invasive front of rapidly proliferating tumors; if this communication can be disrupted and further prevented, this re-formation and invasive capacity should be diminished, providing a unique approach to this cellular problem. There is more attention being paid to consider TNTs/TMs as potential druggable therapeutic targets (46), thus providing another novel potential complementary tactic to treating patients with tumors that are highly invasive and otherwise remain difficult-to-treat using current strategies $(34,35)$.

"If your enemy is secure at all points, be prepared for him. If he is in superior strength, evade him. If your opponent is temperamental, seek to irritate him. Pretend to be weak, that he may grow arrogant. If he is taking ease, give bim no rest."

Cancer is nothing if not temperamental. And arrogant. And superior in its ability to evade so many of the therapeutic modalities we can throw at it, including physical removal through surgery, bombarding it with radiation, chemotherapeutic drugs that target but are not selective for rapidly proliferating cancer cells, and biologic targeted drugs that can bind specifically to identifiable targets and suppress tumor growth but which in many cases do not result in a permanent cure.

There is a wide range of strategies, combinations, and sequencing of multiple therapeutic modalities and the ways they are deployed, and this differs for good reason between different solid tumor types based on known biologic behavior. CRC that metastasize to the liver, a favored site, in limited number are staged as IVA, but in many cases may be considered candidates for surgical resection in combination with chemotherapy, either before and/or after surgery. This combination is used with intent-to-cure, one of the more specific and uncommon circumstances in which a stage IV solid cancer is potentially curable. However, in pancreatic adenocarcinoma, surgery is not recommended for the $~ 50 \%$ of cases that are metastatic at diagnosis, most often to the liver, despite some arguments in favor of this approach (47-49). The reason is that pancreatic and CRC carcinomas are fundamentally different in biologic behavior, and there is enough experience to show that pancreatic carcinomas are so extremely capable of evasion that the potential harm of surgery in these patients far outweighs any potential benefit. What makes these two forms of gastrointestinal cancers different enough that surgical resection is considered reasonable for one, but not for the other? Beyond the behavior of malignant cells in their native tumor microenvironment, more research into understanding how these cells behave once dispersed into the bloodstream and landing in distant sites has provided stimulus for studying these as potential screening, prognostic, and predictive biomarkers of these and other cancers. While the sensitivity and capture rate of CTCs using first-generation devices was rather low-as low as 20-30\% (50)-next-generation technologies have claimed superior capture and the ability to identify and analyze CTCs in as many as $90 \%$ of patients with known diseases. The search for effective biomarkers that can tip off scientists and oncologists alike to identify cancers earlier and to tailor treatments to individual patients via these "liquid biopsies" has many challenges. Biologically, a "self-seeding" hypothesis was proposed that postulated the notion that CTCs circulate systemically and become "educated" by scouting distant metastatic sites (51-53); some of these mature CTCs can then circulate back to "home base"- the primary tumor-and as a result provide impetus for the primary tumor to evolve a drugresistant phenotype. If true, then this hypothesis could explain the somewhat controversial view that surgically resecting a primary tumor in the setting of stage IV malignancy could actually improve overall survival in select populations (54). 


\section{Efforts to improve cancer screening methods and techniques: a step toward preventing the enemy from gaining momentum critical to cancer's success}

"If his forces are united, separate them. If sovereign and subject are in accord, put division between them. Attack him where he is unprepared, appear where you are not expected."-Sun-Tzu

As an example, let's examine one of the most aggressive and difficult-to-treat cancers known to humankind: pancreatic carcinoma. There are an estimated 45,000 new cases of pancreatic cancer in the U.S. each year. This is an especially aggressive and insidious disease for which there is little to no symptomatic forewarning of malignant generation in its earliest stages and no validated screening tool-radiologic, blood-based biomarkers, or otherwisecurrently available for early detection. If an average pancreatic tumor is not diagnosed until 3-6 months after the earliest stages of tumor formation, then the enemy that is cancer has essentially declared war without the host (i.e., the human patient and treatment team) even being aware of this fact until months later, when the cancer is finally diagnosed upon symptomatic presentation. This leaves patients prone to the consequences of an unprovoked attack for which they are unable to mount an appropriate response until the enemy has advanced and breeched the pancreas. Moreover, as $80 \%$ of pancreatic adenocarcinomas present as locally advanced or metastatic disease at the time of diagnosis (55), this form of the disease is essentially defined as an incurable malignancy at the same time the patient gains knowledge of the attack-making both diagnosis and prognosis a doubly devastating message for the patient and family. Even worse, nearly half of patients with forms that are locally advanced (thus not surgically resectable) will manifest frankly metastatic disease within 3 months of diagnosis (56-58). In what other forms of war would we consider it allowable to be caught so off-guard as to be destined to lose the war and to simultaneously be left defenseless without hope for eliminating one's assailants? Evidence is clear that the small minority of patients that have potentially resectable disease have superior prognosis and a small, but real, chance for a cure. If there are ways in which both clinical oncology and medical science can "take the enemy's country whole and intact", while avoiding the ongoing and consequential "shatter and destroy" techniques of modern cancer therapy, which leaves patients suffering from intolerable adverse effects of both disease and treatment, this approach is worth serious examination and more focused efforts.
A recent study from Johns Hopkins University researchers claims to have developed and validated a panel of blood-based markers that can identify cancers for which no effective screening tests are yet available (59). If valid, this provides rich opportunity to learn how to proactively attack dispersed cancer cells and tumors in the earlier stages of carcinogenesis before they can take hold, develop the surrounding microenvironment to their needs, and metastasize.

\section{Knowing when less is more (or, when not to attack)}

"In the practical art of war, the best thing of all is to take the enemy's country whole and intact; to shatter and destroy it is not so good. So, too, it is better to recapture any army entire than to destroy it, to capture a regiment, a detachment or a company entire than to destroy them."-Sun-Tzu

For many years, surgery remained the lynchpin for treatment with intent-to-cure of many solid tumor malignancies that displayed a high rate of eventual recurrence. In the past few decades, trials of post-operative (adjuvant) chemotherapy in which the goal is to eradicate, or at least suppress, residual microscopic disease have shown improvements in overall survival and established new standards of care. A next-wave approach incorporated use of upfront (neoadjuvant) chemotherapy after diagnosis, but prior to surgical resection, with the goal of shrinking the tumor, attacking microscopic disease earlier rather than waiting weeks, or even months, after diagnosis and surgery. Another goal is to ensure that patients receive at least some form of systemic treatment, a point that is especially poignant in cases that require extensive potentially morbid surgery, the complications of which may preclude safe administration of chemotherapy. This approach has been increasingly used to treat patients with breast, ovarian, pancreatic, colorectal, and other cancers in the past decade. There is always the question of which regimen or combination of drugs would be most effective. In examination of trials over time, a "more is better" approach has been employed many times; aside from a potential increase in toxicity, using more non-selective drugs does not necessarily mean a better outcome. For example, the use of a triple-drug regimen comprising cisplatinum, 5-fluorouracil, and an anthracycline (epirubicin) administered for three months prior to and again after surgical resection of gastroesophageal malignancies emerged as a new standard-of-care 10 years ago (60). Until the turn 
of the century, surgery alone remained the most common treatment approach, but with high rates of failure marked by cancer recurrence. Epirubicin in particular carries a strong concern for inducing cardiomyopathy in patients, and has to be used with caution, if at all, in patients with underlying cardiac dysfunction. However, more recent studies seem to dispel the belief that adding this 3rd drug has any benefit at all; as a result, the use of epirubicin has strongly fallen out of favor, with the idea that in this context more is definitely not better, and can in fact be significantly worse. Another prime example of how the paradigm has shifted away from a "one size fits all" approach to patients with the same histopathologic diagnosis comes from the breast cancer world (61). It is well-established that breast cancer is a diverse set of malignant tumors subtyped histopathologically and by molecular expression of hormone receptors (estrogen and progesterone receptors) and the EGFR HER2. As it is known that some patients with estrogen-receptor (ER)positive, lymph-node negative breast cancer derived little to no benefit from chemotherapy, compared to other subtypes, a 21-gene recurrence score was devised to better stratify which patients would indeed benefit, and which patients could be spared unnecessary toxicity as their tumors would be unlikely to respond. The scoring system is now widely used in the clinic to quantify risk of 10 -year distant recurrence by category (low, <18; intermediate, 18 to 30 ; high, $\geq 31$ ) for treatment management among women diagnosed with hormone receptor-positive, human EGFR 2 -negative, lymph node-negative breast cancer $(61,62)$.

\section{Conclusions and developing thoughts}

Lessons taken from history can be applied to both basic scientific cancer research as well as the clinical practice of oncology, with the goal of elevating paradigms for cancer treatment to greater success. The overall foundation of knowledge of cancer biology has increased exponentially in a relatively short amount of time. Excitement is warranted, but the importance of critical analysis and context cannot be underestimated. With questions of reproducibility of data originating in studies performed both in vitro and in vivo in animal models (data that often serve as the rationale for clinical trials in human patients) (63), it is important that scientists and clinicians think carefully and deliberately about how we interpret such results and bring them forward "from bench to bedside" in as rational a manner as possible. The term "bench-to-bedside" is a popular but cliché catchphrase since at least the 1990's.
However, this paradigm assumes that the problem-solving approach should be initiated in the laboratory as opposed to the source of the problem: the cancer inside human patients. To truly know the enemy that is cancer, rational approaches include studying and understanding the human condition in cancer patients and identifying specific trends-both positive and negative - that can be elucidated at the molecular and cellular levels in the laboratory. Such an approach requires more of a "bedside-to-bench-andthen-back-to-bedside" mindset that, while used less widely as a catchphrase, more accurately reflects a rational strategy that ensures clinically relevant approaches are taken in research. The success of this approach is predicated upon a true translational approach based on team science, in which clinical oncology teams make accurate diagnostic assessment and work closely with basic science teams who have the expertise in established molecular pathways and the capability to identify new pathways to explain new clinical observations. Examples of this approach are becoming more prevalent with rising interest in "n-of-1" approaches that examine molecular genetics and profiles of best responders or conversely of non-responders to targeted cancer therapies.

The art of the medical practice of oncology necessitates a complex balance between adherence to and scrutiny of evidence-based medicine and clinical judgment. The art of war (embodied by The Art of War) similarly necessitates a formulaic approach to application and adaptation of strategies and tactics taken in tandem. In the burgeoning era of advanced genomics, identification of new forms of biomarkers for assessment of diagnosis and prognosis, rational molecular targets for therapy, and most especially awareness of the need for team science approaches to translation into the clinic, it is essential that the pathway to drug development be paved with understanding and seamless transitions from bedside to bench and back again. True success in this case will not be measured by the number of published papers in high-impact journals, monetary gain from sales of drugs, or from clinical revenue. Success can only be measured by appropriate and accurate translation of laboratory and translational research findings into preventing and treatment-directed applications designed to improve the human condition, either to prevent cancer, to catch it early enough to assure successful attempts at intent-to-cure treatment, and to tailor treatments to individual patients with advanced cancers in order to maximize success as measured by minimizing impact of cancer on their quality of life. 


\section{Acknowledgements}

The author wishes to thank innumerable cancer research and oncology colleagues who through the years have provided helpful discussion that stimulated formulation of thoughts for this article.

Funding: Research in the Lou laboratory has been generously supported by a NIH Clinical and Translational Science KL2 Scholar Award (8UL1TR000114); The Central Society for Clinical and Translational Research Early Career Development Award; The Randy Shaver Cancer Research and Community Fund; the Litman Family Fund for Cancer Research; National Pancreas Foundation Research Grant (provided in partnership with the National Pancreas Foundation, several NPF Chapters and the Horvitz/Lebovitz Research Fund); the Mezin-Koats Colon Cancer Research Award; the University of Minnesota Deborah E. Powell for Women's Health Interdisciplinary Seed Grant support (Grant \#PCWH-2013-002); Institutional Research Grant \#118198-IRG-58-001-52IRG94 from the American Cancer Society; Minnesota Masonic Charities; Minnesota Medical Foundation/ University of Minnesota Foundation; and the Masonic Cancer Center and Department of Medicine, Division of Hematology, Oncology and Transplantation, University of Minnesota. The content is solely the responsibility of the author and does not necessarily represent the official views of the National Institutes of Health.

\section{Footnote}

Conflicts of Interest: The author has no conflicts of interest to declare.

\section{References}

1. Silverberg E, Holleb AI. Cancer statistics, 1971. CA Cancer J Clin 1971;21:13-31.

2. Siegel RL, Miller KD, Jemal A. Cancer Statistics, 2017. CA Cancer J Clin 2017;67:7-30.

3. Kruijff S, Van Zweden C. The harmful impact of the rhetoric "war on cancer". Eur J Surg Oncol 2017;43:963-4.

4. Oronsky BT, Carter CA, Oronsky AL, et al. "No patient left behind": an alternative to "the War on Cancer" metaphor. Med Oncol 2016;33:55.

5. Haines I. The war on cancer: time for a new terminology. Lancet 2014;383:1883.

6. Ellis LM, Blanke CD, Roach N. Losing "Losing the Battle
With Cancer". JAMA Oncol 2015;1:13-4.

7. Hingorani SR, Zheng L, Bullock AJ, et al. HALO 202: Randomized Phase II Study of PEGPH20 Plus Nab-Paclitaxel/Gemcitabine Versus Nab-Paclitaxel/ Gemcitabine in Patients With Untreated, Metastatic Pancreatic Ductal Adenocarcinoma. J Clin Oncol 2018;36:359-66.

8. Sottoriva A, Spiteri I, Piccirillo SG, et al. Intratumor heterogeneity in human glioblastoma reflects cancer evolutionary dynamics. Proc Natl Acad Sci U S A 2013;110:4009-14.

9. Thress KS, Paweletz CP, Felip E, et al. Acquired EGFR C797S mutation mediates resistance to AZD9291 in nonsmall cell lung cancer harboring EGFR T790M. Nat Med 2015;21:560-2.

10. Yanagita M, Redig AJ, Paweletz CP, et al. A Prospective Evaluation of Circulating Tumor Cells and Cell-Free DNA in EGFR-Mutant Non-Small Cell Lung Cancer Patients Treated with Erlotinib on a Phase II Trial. Clin Cancer Res 2016;22:6010-20.

11. Fukuoka $M, W u$ YL, Thongprasert S, et al. Biomarker analyses and final overall survival results from a phase III, randomized, open-label, first-line study of gefitinib versus carboplatin/paclitaxel in clinically selected patients with advanced non-small-cell lung cancer in Asia (IPASS). J Clin Oncol 2011;29:2866-74.

12. Mok TS, Wu YL, Thongprasert S, et al. Gefitinib or carboplatin-paclitaxel in pulmonary adenocarcinoma. $\mathrm{N}$ Engl J Med 2009;361:947-57.

13. Mok TS, Wu YL, Ahn MJ, et al. Osimertinib or PlatinumPemetrexed in EGFR T790M-Positive Lung Cancer. N Engl J Med 2017;376:629-40.

14. Mok TS, Wu YL, Papadimitrakopoulou VA. Osimertinib in EGFR T790M-Positive Lung Cancer. N Engl J Med 2017;376:1993-4.

15. Bivona TG, Doebele RC. A framework for understanding and targeting residual disease in oncogene-driven solid cancers. Nat Med 2016;22:472-8.

16. Loree JM, Pereira AA, Lam M, et al. Classifying colorectal cancer by tumor location rather than sidedness highlights a continuum in mutation profiles and Consensus Molecular Subtypes. Clin Cancer Res 2018;24:1062-72.

17. Yaeger R, Cercek A, Chou JF, et al. BRAF mutation predicts for poor outcomes after metastasectomy in patients with metastatic colorectal cancer. Cancer 2014;120:2316-24.

18. Yang H, Higgins B, Kolinsky K, et al. Antitumor activity of BRAF inhibitor vemurafenib in preclinical 
models of BRAF-mutant colorectal cancer. Cancer Res 2012;72:779-89.

19. Kopetz S, Desai J, Chan E, et al. Phase II Pilot Study of Vemurafenib in Patients With Metastatic BRAF-Mutated Colorectal Cancer. J Clin Oncol 2015;33:4032-8.

20. Prahallad A, Sun C, Huang S, et al. Unresponsiveness of colon cancer to BRAF(V600E) inhibition through feedback activation of EGFR. Nature 2012;483:100-3.

21. Mao M, Tian F, Mariadason JM, et al. Resistance to BRAF inhibition in BRAF-mutant colon cancer can be overcome with PI3K inhibition or demethylating agents. Clin Cancer Res 2013;19:657-67.

22. Corcoran RB, Atreya CE, Falchook GS, et al. Combined BRAF and MEK Inhibition With Dabrafenib and Trametinib in BRAF V600-Mutant Colorectal Cancer. J Clin Oncol 2015;33:4023-31.

23. Hong DS, Morris VK, El Osta B, et al. Phase IB Study of Vemurafenib in Combination with Irinotecan and Cetuximab in Patients with Metastatic Colorectal Cancer with BRAFV600E Mutation. Cancer Discov 2016;6:1352-65.

24. Stamelos VA, Robinson E, Redman CW, et al. Navitoclax augments the activity of carboplatin and paclitaxel combinations in ovarian cancer cells. Gynecol Oncol 2013;128:377-82.

25. Xiong X, Sui M, Fan W, et al. Cell cycle dependent antagonistic interactions between paclitaxel and carboplatin in combination therapy. Cancer Biol Ther 2007;6:1067-73.

26. Leder K, Pitter K, LaPlant Q, et al. Mathematical modeling of PDGF-driven glioblastoma reveals optimized radiation dosing schedules. Cell 2014;156:603-16.

27. Messenheimer DJ, Jensen SM, Afentoulis ME, et al. Timing of PD-1 Blockade Is Critical to Effective Combination Immunotherapy with Anti-OX40. Clin Cancer Res 2017;23:6165-77.

28 Lu YS, Chen TW, Lin CH, et al. Bevacizumab preconditioning followed by Etoposide and Cisplatin is highly effective in treating brain metastases of breast cancer progressing from whole-brain radiotherapy. Clin Cancer Res 2015;21:1851-8.

29. Hurwitz HI, Yi J, Ince W, et al. The clinical benefit of bevacizumab in metastatic colorectal cancer is independent of K-ras mutation status: analysis of a phase III study of bevacizumab with chemotherapy in previously untreated metastatic colorectal cancer. Oncologist 2009;14:22-8.

30. Hurwitz H, Fehrenbacher L, Novotny W, et al. Bevacizumab plus irinotecan, fluorouracil, and leucovorin for metastatic colorectal cancer. N Engl J Med 2004;350:2335-42.

31. Allegra CJ, Yothers G, O'Connell MJ, et al. Phase III trial assessing bevacizumab in stages II and III carcinoma of the colon: results of NSABP protocol C-08. J Clin Oncol 2011;29:11-6.

32. Hadad SM, Coates P, Jordan LB, et al. Evidence for biological effects of metformin in operable breast cancer: biomarker analysis in a pre-operative window of opportunity randomized trial. Breast Cancer Res Treat 2015;150:149-55.

33. Niraula S, Dowling RJ, Ennis M, et al. Metformin in early breast cancer: a prospective window of opportunity neoadjuvant study. Breast Cancer Res Treat 2012;135:821-30.

34. Lou E. Can you hear them now? Tumor microtubes form cellular communication networks that protect gliomas from surgical lesions and chemotherapy treatments. Neuro Oncol 2017;19:1289-91.

35. Marlein CR, Zaitseva L, Rushworth SA. Pulling the plug - halting cancer's theft of mitochondria. Oncoscience 2017;4:173-4.

36. Costa-Silva B, Aiello NM, Ocean AJ, et al. Pancreatic cancer exosomes initiate pre-metastatic niche formation in the liver. Nat Cell Biol 2015;17:816-26.

37. Peinado H, Aleckovic M, Lavotshkin S, et al. Melanoma exosomes educate bone marrow progenitor cells toward a pro-metastatic phenotype through MET. Nat Med 2012;18:883-91.

38. Weil S, Osswald M, Solecki G, et al. Tumor microtubes convey resistance to surgical lesions and chemotherapy in gliomas. Neuro Oncol 2017;19:1316-26.

39. Osswald M, Jung E, Sahm F, et al. Brain tumour cells interconnect to a functional and resistant network. Nature 2015;528:93-8.

40. Ariazi J, Benowitz A, De Biasi V, et al. Tunneling Nanotubes and Gap Junctions-Their Role in LongRange Intercellular Communication during Development, Health, and Disease Conditions. Front Mol Neurosci 2017;10:333.

41. Lou E, O'Hare P, Subramanian S, et al. Lost in translation: applying 2D intercellular communication via tunneling nanotubes in cell culture to physiologically relevant 3D microenvironments. FEBS J 2017;284:699-707.

42. Ady JW, Desir S, Thayanithy V, et al. Intercellular communication in malignant pleural mesothelioma: properties of tunneling nanotubes. Front Physiol 2014;5:400. 
43. Lou E, Fujisawa S, Barlas A, et al. Tunneling Nanotubes: A new paradigm for studying intercellular communication and therapeutics in cancer. Commun Integr Biol 2012;5:399-403.

44. Lou E, Fujisawa S, Morozov A, et al. Tunneling nanotubes provide a unique conduit for intercellular transfer of cellular contents in human malignant pleural mesothelioma. PLoS One 2012; 7:e33093.

45. Lou E. Intercellular conduits in tumours: the new social network. Trends Cancer 2016;2:3-5.

46. Baker M. How the Internet of cells has biologists buzzing. Nature 2017;549:322-4.

47. Zanini N, Lombardi R, Masetti M, et al. Surgery for isolated liver metastases from pancreatic cancer. Updates Surg 2015;67:19-25.

48. Yamada H, Hirano S, Tanaka E, et al. Surgical treatment of liver metastases from pancreatic cancer. HPB (Oxford) 2006;8:85-8.

49. Arnaoutakis GJ, Rangachari D, Laheru DA, et al. Pulmonary resection for isolated pancreatic adenocarcinoma metastasis: an analysis of outcomes and survival. J Gastrointest Surg 2011;15:1611-7.

50. Lou E, Vogel RI, Teoh D, et al. Assessment of Circulating Tumor Cells as a Predictive Biomarker of Histology in Women With Suspected Ovarian Cancer. Lab Med 2018;49:134-9.

51. Comen E, Norton L. Self-seeding in cancer. Recent Results Cancer Res 2012;195:13-23.

52. Comen E, Norton L, Massague J. Clinical implications of cancer self-seeding. Nat Rev Clin Oncol 2011;8:369-77.

53. Kim MY, Oskarsson T, Acharyya S, et al. Tumor selfseeding by circulating cancer cells. Cell 2009;139:1315-26.

54. Gresham G, Renouf DJ, Chan M, et al. Association between palliative resection of the primary tumor and overall survival in a population-based cohort of metastatic colorectal cancer patients. Ann Surg Oncol 2014;21:3917-23.

Cite this article as: Lou E. The Art of War and oncology: applying the principles of strategy and tactics to greater effect in the era of targeted therapy. Ann Transl Med 2018;6(9):168. doi: 10.21037/atm.2018.03.04
55. Zhang Q, Zeng L, Chen Y, et al. Pancreatic Cancer Epidemiology, Detection, and Management. Gastroenterol Res Pract 2016;2016:8962321.

56. Mukherjee S, Hurt CN, Bridgewater J, et al. Gemcitabinebased or capecitabine-based chemoradiotherapy for locally advanced pancreatic cancer (SCALOP): a multicentre, randomised, phase 2 trial. Lancet Oncol 2013;14:317-26.

57. Hurt CN, Mukherjee S, Bridgewater J, et al. HealthRelated Quality of Life in SCALOP, a Randomized Phase 2 Trial Comparing Chemoradiation Therapy Regimens in Locally Advanced Pancreatic Cancer. Int J Radiat Oncol Biol Phys 2015;93:810-8.

58. Balaban EP, Mangu PB, Yee NS. Locally Advanced Unresectable Pancreatic Cancer: American Society of Clinical Oncology Clinical Practice Guideline Summary. J Oncol Pract 2017;13:265-9.

59. Cohen JD, Li L, Wang Y, et al. Detection and localization of surgically resectable cancers with a multi-analyte blood test. Science 2018;359:926-30.

60. Cunningham D, Allum WH, Stenning SP, et al. Perioperative chemotherapy versus surgery alone for resectable gastroesophageal cancer. $\mathrm{N}$ Engl J Med 2006;355:11-20.

61. Jasem J, Amini A, Rabinovitch R, et al. 21-Gene Recurrence Score Assay As a Predictor of Adjuvant Chemotherapy Administration for Early-Stage Breast Cancer: An Analysis of Use, Therapeutic Implications, and Disparity Profile. J Clin Oncol 2016;34:1995-2002.

62. Jasem J, Fisher CM, Amini A, et al. The 21-Gene Recurrence Score Assay for Node-Positive, Early-Stage Breast Cancer and Impact of RxPONDER Trial on Chemotherapy Decision-Making: Have Clinicians Already Decided? J Natl Compr Canc Netw 2017;15:494-503.

63. Begley CG, Ellis LM. Drug development: Raise standards for preclinical cancer research. Nature 2012;483:531-3. 